\title{
The role of 3-D geomagnetic induction in the determination of the ionospheric currents from the ground geomagnetic data
}

\author{
A. Pulkkinen ${ }^{1}$ and M. Engels ${ }^{2}$ \\ ${ }^{1}$ NASA/GSFC, Greenbelt, Code 692, MD 20770, USA \\ ${ }^{2}$ Federal Institute for Geosciences and Natural Resources, Stilleweg 2, 30655 Hannover, Germany \\ Received: 25 August 2004 - Revised: 13 January 2005 - Accepted: 27 January 2005 - Published: 30 March 2005
}

\begin{abstract}
The geomagnetic field variations measured at the surface of the Earth are composed of both internal and external parts. The external field arises from the sources in the magnetosphere and ionosphere, whereas the internal field is generated by the currents induced within the Earth. The internal part may in some situations comprise a notable part of the measured total field and thus a blind usage of geomagnetic field recordings potentially produces significant errors to estimated ionospheric currents. In this paper the role of geomagnetic induction in auroral ionospheric studies is investigated by modeling the induction using simultaneously the realistic ionospheric source and a realistic three-dimensional Earth conductivity structure.

The modeling results imply that the effects of the lateral ground conductivity anomalies on ionospheric equivalent current patterns are, though clearly detected, less severe than anticipated for fields varying with periods from 5 to $120 \mathrm{~min}$. However, the amplification of the determined currents caused by induction is significant, leading to an overestimation of up to $30 \%$ of the main current flow intensities, with the overestimation increasing sharply when moving away from the region of the main flow.

In addition to the 3-D modeling, a simple method is introduced to help estimate the internal contribution to the measured variations of the $I L$ index (local variant of the $A L$ index). A test with the 26 June 1998 substorm event indicates that the method can help to extract the internal contribution from the $I L$ index.
\end{abstract}

Keywords. Geomagnetism and paleomagnetism (Geomagnetic induction)

\section{Introduction}

The magnetic effect arising from electromagnetic induction inside the Earth contributes to the total magnetic field measured at the surface of the Earth. Depending on the indi-

Correspondence to: A. Pulkkinen

(antti.pulkkinen@gsfc.nasa.gov) vidual situation and the accuracy with which the conclusions are drawn from the data, it may be acceptable to disregard the internal effects. For example, in ionospheric studies the effects of the induction are often neglected. Studies where the internal effects are negligible include, in particular, investigations of temporally relatively steady current systems that do not induce notable currents in the Earth - induction is a time dependent phenomenon. However, when more dynamic situations are concerned, neglecting the induction may lead to erroneous interpretations. In some cases, for example in particular solid Earth studies, separated field components are needed as a starting point for the entire investigation (e.g. Berdichevsky and Zhdanov, 1984, p. 191). Earlier investigations have shown that neglecting telluric effects may cause significant errors of up to $40 \%$ in the estimation of the ionospheric current intensity (Viljanen et al., 1995; Tanskanen et al., 2001), or even notable errors in the determination of some global magnetospheric indices like $D_{s t}$ (Häkkinen et al., 2002). As finer spatial and temporal scales are investigated both in ionospheric and magnetospheric studies and more accurate estimates of the physical parameters are pursued, errors of $40 \%$ become significant and cannot be blindly neglected. It is thus clear that geomagnetic induction should be taken into account in detailed studies of ionospheric and magnetospheric dynamics and further understanding of the distorting effects of geomagnetic induction, especially in regions with strong conductivity gradients, is needed.

Earlier efforts on the modeling of geomagnetic induction have been limited in terms of two typical approximations. In solid Earth-oriented studies, where the complex ground conductivity structures may have been utilized, simplified ionospheric source fields, typically a plane wave, have been used (e.g. Kaikkonen, 1998; Beamish et al., 2002). However, also non-planar source effects have been considered (e.g., Mareschal, 1986; Engels et al., 2002). On the other hand, in space-oriented studies, where more realistic sources have been applied, the ground conductivity structures have been fairly simple, typically one-dimensional (e.g. Pirjola, 1982; Viljanen et al., 1999). Taking into account the complexity of the auroral ionospheric current systems (e.g. Kamide 


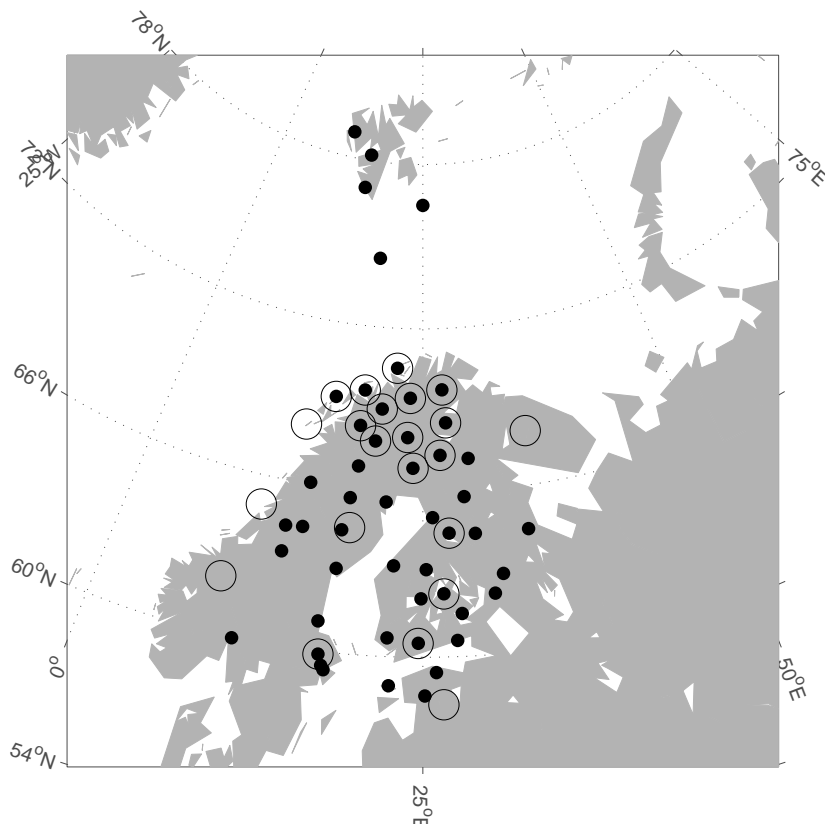

Fig. 1. Location of magnetometer sites used for the derivation of the original source currents (black dots) and the location of sites used for the derivation of the currents used in the comparison (circles). Geographic coordinates are used.

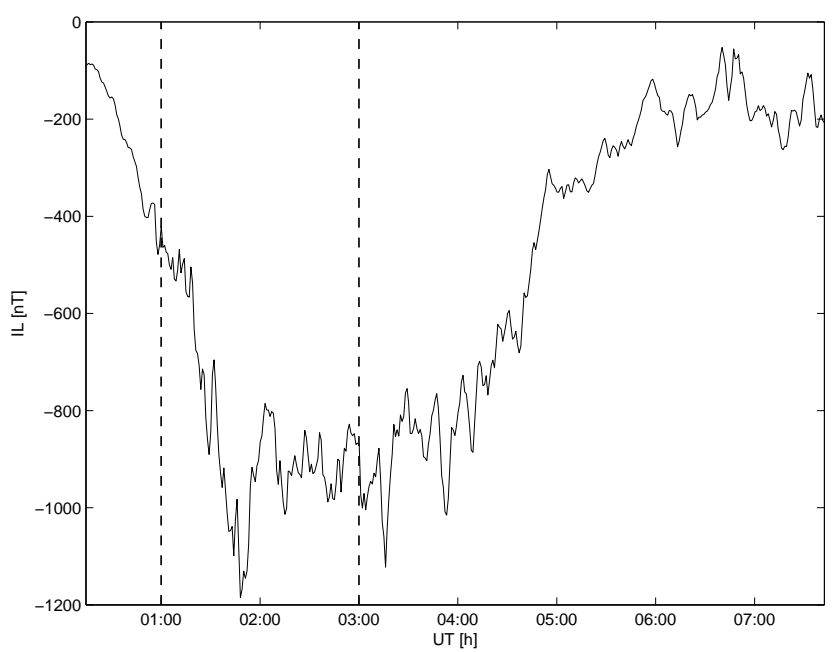

Fig. 2. The local variant of $A L, I L$ index computed from the measured IMAGE magnetometer array data for 26 June 1998. The dashed lines indicate 01:00 UT and 03:00 UT, respectively.

and Baumjohann, 1993) and the non-one-dimensional nature of the Earth's crust (e.g. Heinson, 1999, and references therein), the approximations seem quite crude, thus imposing limitations to the conclusions drawn from these earlier investigations. Here, for the first time, a realistic threedimensional (3-D) model of the ground conductivity is combined with a geometrically varying and arbitrarily moving realistic ionospheric source. Comparisons between the original ionospheric source and the ionospheric source deduced using the ground magnetic field "contaminated" by the modeled induction enables us to estimate the effect of the induction more realistically than in earlier investigations. Olsen and Kuvshinov (2004) have used a similar approach to study the coast effect of geomagnetic storms, i.e. a global conductivity model was excited with magnetic field variations produced by the ring current. Their study, however, due to its global nature, is not directly comparable to the regional study carried out here.

In Sect. 2, we describe the modeling procedure that enables the combination of the 2-D ionospheric source and 3-D geomagnetic induction. In Sect. 3, we carry out a detailed analysis of the modeled data and the impact of 3-D induction on the determined ionospheric equivalent currents is estimated. Also, a new, simple method for estimating the internal effects on geomagnetic indices, like $I L$ (the local variant of the $A L$ index computed from the IMAGE magnetometer array data; see Kauristie et al. (1996)), is introduced. The theoretical basis of the method is given in the Appendix. Finally, in Sect. 4, summary and concluding remarks with general implications of the study are given.

\section{Modeling procedure}

A volume-3-D induction code by Avdeev et al. (2002), combined with the new field separation technique introduced by Pulkkinen et al. (2003b), provide efficient tools for the treatment given in the paper. The four-step modeling procedure is as follows:

1. Data from the Baltic Electromagnetic Array Research (BEAR) project during which both the electric and magnetic field were recorded in a dense array covering Fennoscandia (Korja et al., 2002), combined with IMAGE magnetometer array data, are used (black dots in Fig. 1). First, the geomagnetic data are separated into internal and external parts to obtain the best possible estimate of the ionospheric equivalent source (Pulkkinen et al., 2003b).

2. A two-hour event from 26 June 1998, 01:00-03:00 UT, part of a relatively intense substorm event (see Fig. 2), was selected for 3-D modeling in the frequency domain. Code inputs are the external source model and the internal conductivity Earth model. Step one provides the ionospheric equivalent current systems in time domain, which are Fourier transformed to frequency domain and represented by electrical dipoles after projection and interpolation in the modeling grid at $110 \mathrm{~km}$ height. The Fennoscandian conductivity model is based on the conductance maps by Korja et al. (2002). The 3-D crust consists of 8 inhomogeneous layers increasing in thickness with depth and representing the upper $60 \mathrm{~km}$ (see Fig. 3). Below, a layered 1-D Earth model describes the normal structure of the Fennoscandian shield according to Varentsov et al. (2002) (Table 3, ENG model without asthenosphere). In Varentsov et al. (2002) the 


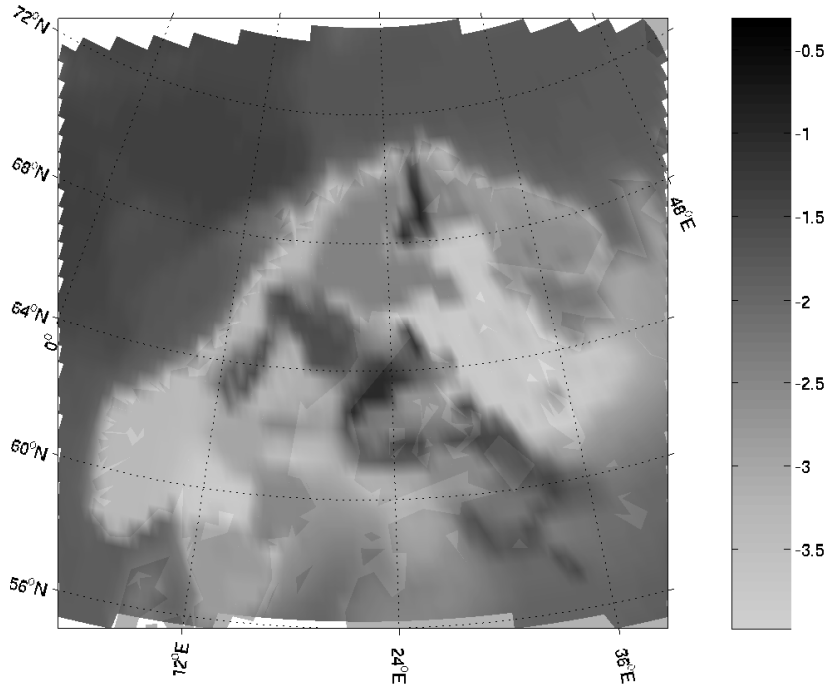

Fig. 3. $\log _{10}$ of the average taken over the Fennoscandian crustal $(0-60 \mathrm{~km})$ conductivity $[\mathrm{S} / \mathrm{m}]$ model used in the volume-3-D induction code.

construction of the 3-D model is also described in detail. Modeling frequencies are 24 harmonics of the selected two-hour event, resulting in a period range from 5 to $120 \mathrm{~min}$. All model cells have a $20-\mathrm{km}$ base length and the total modeling area extends to $168 \times 180$ cells $(3360 \mathrm{~km} \times 3600 \mathrm{~km})$, surrounded by the 1 -D normal section.

3. The output of the modeling code consists of the electromagnetic fields for each modeling cell in frequency domain. After inverse Fourier transformation back into time domain, two magnetic data sets are extracted for the continental IMAGE magnetometer locations (circles in Fig. 1): one with the total and another with the external part of the magnetic field. The northernmost IMAGE sites are not applicable due to their vicinity to the volume-3-D modeling boundary.

4. From the two sets of magnetic field data, we derive two sets of ionospheric equivalent currents (on equivalent currents, see e.g., Untiedt and Baumjohann, 1993), using the spherical elementary current system method (Amm and Viljanen, 1999; Pulkkinen et al., 2003a). The currents are derived to $110 \mathrm{~km}$ height. Only the horizontal components of the magnetic field are used. This is due to the fact that the horizontal field can always be explained by a source only in the ionosphere, or in the ground (for a more detailed discussion, see Pulkkinen et al., 2003a). Thus, the vertical component of the magnetic field is not treated in this investigation. Finally, the difference between the two sets of equivalent currents is analyzed and the impact of induction in the ground on the equivalent currents is estimated.
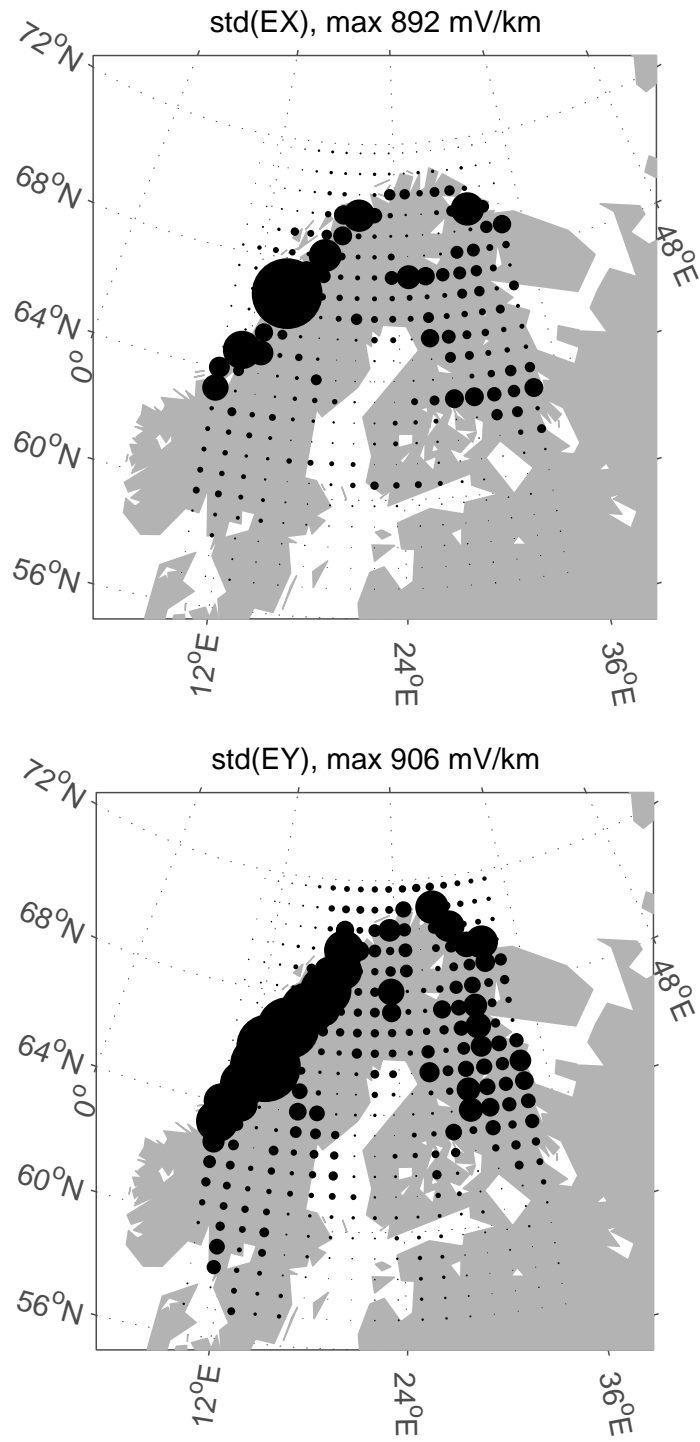

Fig. 4. Standard deviation of the modeled geoelectric field variations at the Earth's surface at some of the grid points of the volume3-D code. The maximum standard deviations are indicated in the titles of the figures.

\section{Analysis}

Let us first see if the modeled fields have features arising from the non-uniform ground conductivity. We also check if the modeled total magnetic field corresponds to the observational data. Figure 4 shows the standard deviation taken over the time series of the modeled surface geoelectric field at selected grid points of the volume-3-D code. The coastline effect, driven by the large conductivity contrast between the sea and the continent (e.g. Beamish et al., 2002), causes very large electric fields variations. Also, other inhomogeneities are seen, for example, notably smaller surface electric fields are obtained at the diagonal well-conducting band going across the modeling area. In general, variations in standard deviations clearly coincide with the inhomogeneities of 

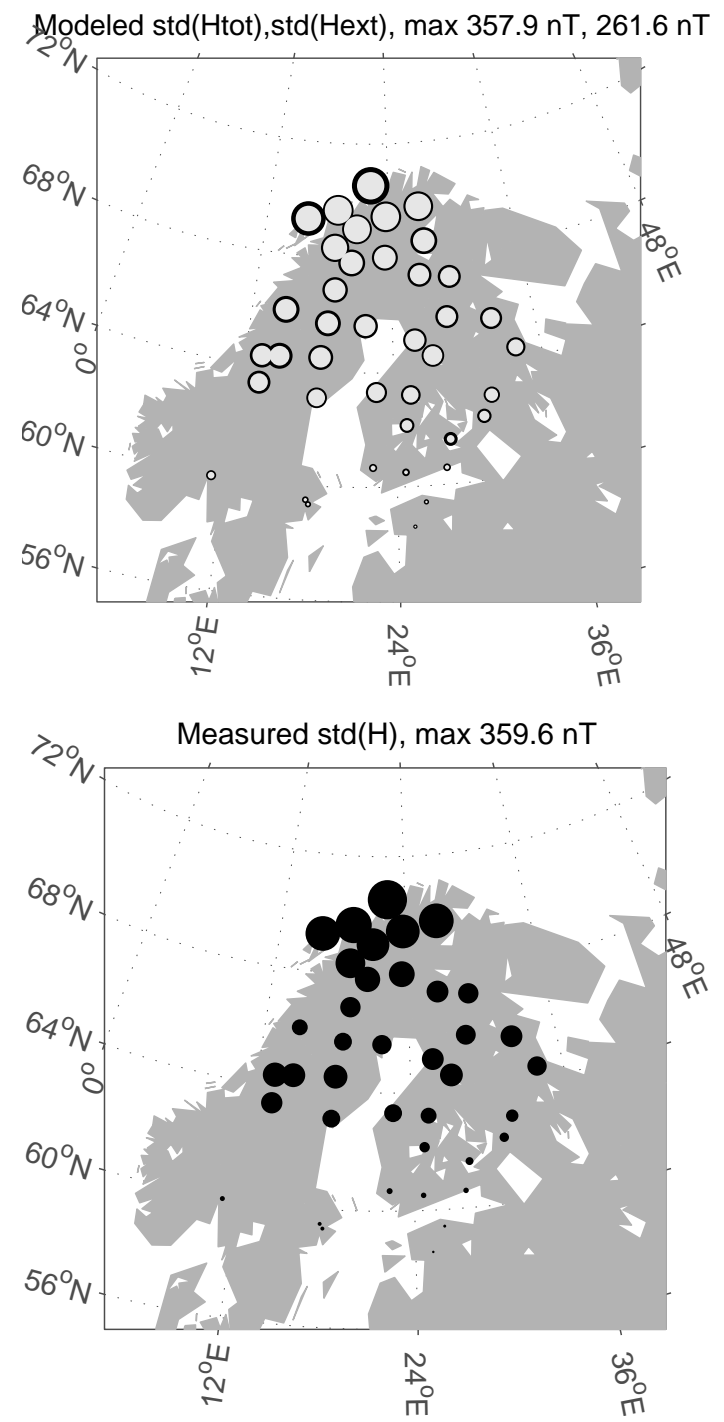

Fig. 5. Standard deviations of the modeled and the measured horizontal magnetic field variations. Light and black circles on the top panel correspond to standard deviations of the external field and the total field, respectively. The maximum standard deviations are indicated in the titles of the figures.

the ground. Figure 5, in turn, shows the modeled and measured standard deviations of the horizontal magnetic field. The measured data was low-pass filtered to contain only periods of $5 \mathrm{~min}$ and above, to correspond to the periods included in the modeled data. It is seen that the magnitudes of the standard deviations and the spatial patterns of the total modeled and the measured field variations coincide very well with each other. Furthermore, as seen by comparing the external and the total modeled magnetic field variations, induced fields amplify the external field variations, as expected. Finally, as can be verified by comparing Figs. 6 and 13 , the modeled and the measured 5-min low-pass filtered $I L$ index, respectively, the modeled $I L$ coincides well with the measurements, yet there is another indication of a realistic Earth response. Thus, it can be concluded that the modeling
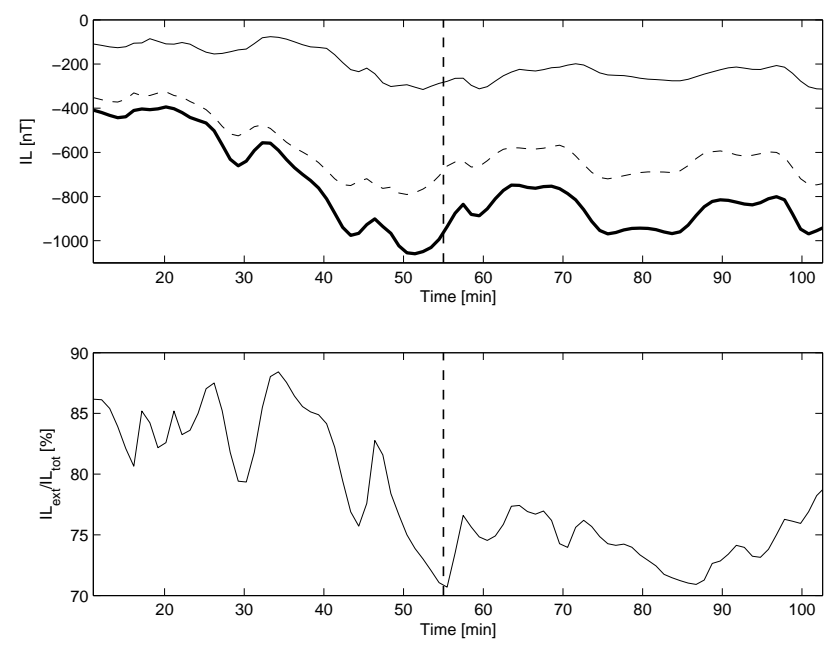

Fig. 6. Top panel: modeled external (dashed), internal (thin solid) and the total $I L$ (thick solid) index. bottom panel: ratio between the external $I L$ and the total $I L$. time is minutes from the beginning of the modeling period of 26 June 1998, 01:00 UT. The dashed line indicates the time 01:55 UT.

procedure produces both 3-D and an realistic Earth response, and thus the modeled fields can be assumed to reflect the true characteristics of the investigated phenomenon.

The question we aim to answer is "How much does geomagnetic induction in a realistic 3-D Earth affect the derived ionospheric equivalent current magnitudes and their spatial patterns?". The question is addressed in Figs. 6-13. In Fig. 6 we show the contribution of the internal and external modeled magnetic fields to the $I L$ index. Note that due to a $20 \%$ tapering of the source data prior to the Fourier transformation, 10-15 min from both ends of the modeling period are not usable in the analysis. In general, excluding about a $5 \%$ difference in the peak internal contribution to $I L$, the results presented in Fig. 6 are very similar to those in Fig. 7 of Pulkkinen et al. (2003b), where the separated components of the measured $I L$ for the same substorm event are depicted, another indication of realistic modeled fields. The low-frequency component of the internal part of $I L$ starts to gradually increase from the time of the substorm onset, reaching the maximum roughly at the end of the expansion phase when the internal part comprises about $30 \%$ of the observed signal. After the expansion phase, the internal part starts to gradually decrease. An important observation to make from Fig. 6 is that the ratio $I L_{e x t} / I L_{t o t}$ also has the high-frequency component and that the relative contributions of external and internal parts can vary quite strongly around the average, a feature also seen clearly in Pulkkinen et al. (2003b). We note that the peak internal contribution of $30 \%$ to the total $I L$ is about $10 \%$ smaller than what was reported by Tanskanen et al. (2001) for the average over a number of substorms, and, as mentioned above, $5 \%$ smaller than that reported by Pulkkinen et al. (2003b). This may indicate that the conductivity model is slightly too resistive. Here a 1-D model without the asthenosphere was utilized; 


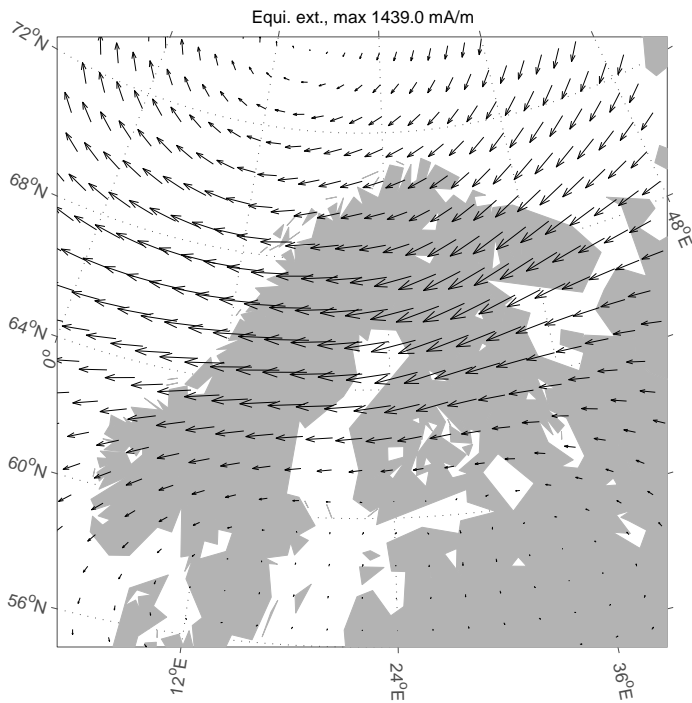

Fig. 7. Ionospheric equivalent currents $\left(\boldsymbol{J}_{\text {ext }}\right)$ determined from the external part of the modeled magnetic field. Magnetic field data from the continental IMAGE array locations for time 01:55 UT are used (see Fig. 6). The maximum current amplitude is indicated in the title of the figure.

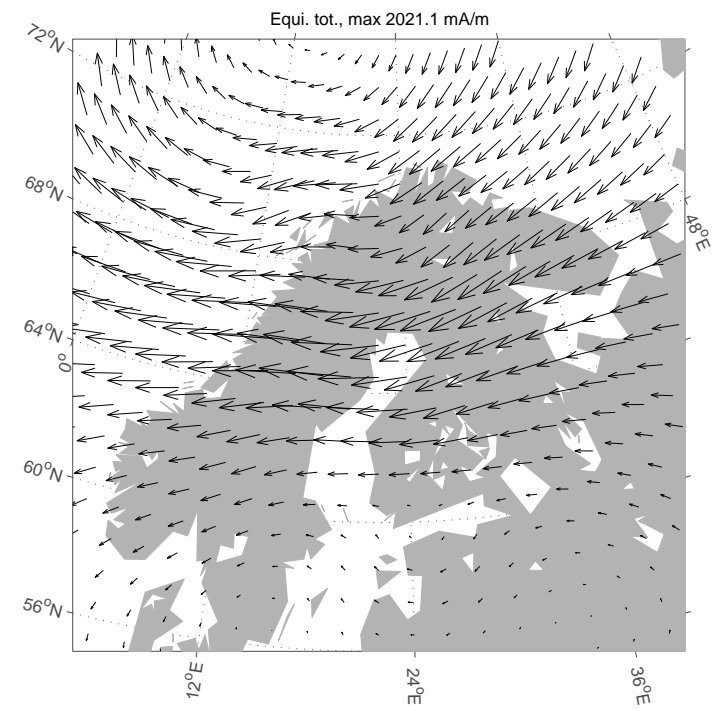

Fig. 8. Ionospheric equivalent currents $\left(\boldsymbol{J}_{\text {tot }}\right)$ determined from the total modeled magnetic field. Magnetic field data from the continental IMAGE array locations for time 01:55 UT are used (see Fig. 6). The maximum current amplitude is indicated in the title of the figure.

the asthenosphere variant with an increased conductance at 200-300 km depth would enhance the internal contribution on the order of $5-10 \%$. However, the usage of a slightly too resistive conductivity model does not affect the basic conclusions of the study.

In Figs. 7-10 we show a snapshot of the derived ionospheric equivalent currents and their relative differences for the time when the peak internal contribution to $I L$ occurs.

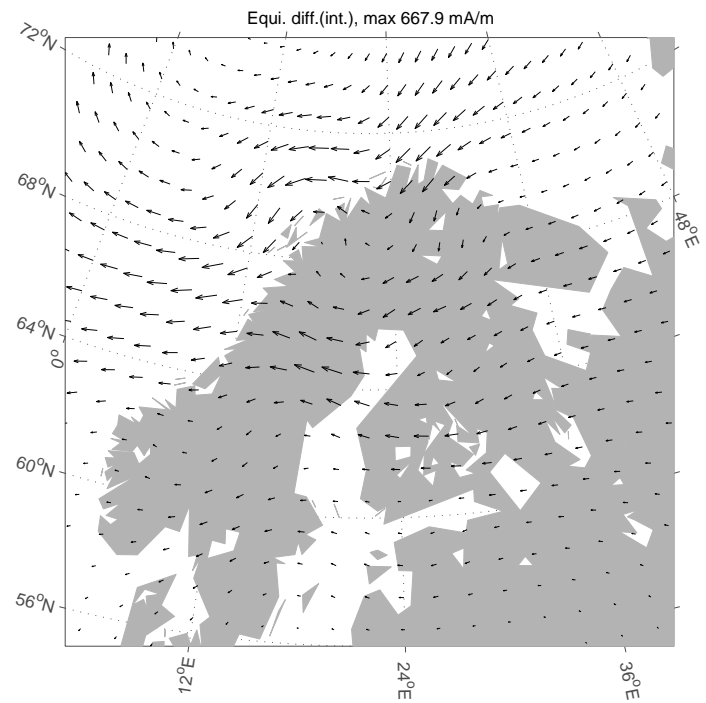

Fig. 9. Difference between the ionospheric equivalent currents determined from the total and external parts of the modeled magnetic field. Magnetic field data from the continental IMAGE array locations for time 01:55 UT are used (see Fig. 6). The maximum current amplitude (difference) is indicated in the title of the figure.

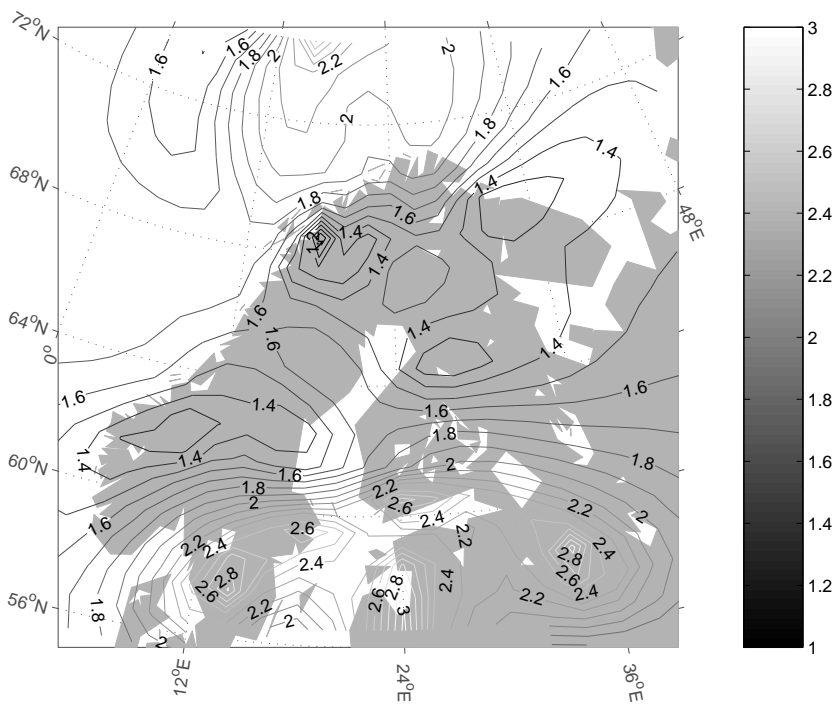

Fig. 10. $\log _{10}$ of the relative difference $100 \cdot\left|\boldsymbol{J}_{\text {tot }}-\boldsymbol{J}_{\text {ext }}\right| /\left|\boldsymbol{J}_{\text {ext }}\right|$. See Figs. 7-9.

Although not shown here, the view is also very similar to earlier times and later to the depicted time. The very basic feature is the amplification of the equivalent currents due to induction. As already seen from Fig. 6, an overestimation of about $30 \%$ of the current amplitude is made in the region of the main flow. As we move outside the region of the main flow, the overestimation increases sharply to $100 \%$ and more. This is explained by the fact that the induced currents are distributed latitudinally to a wider area than the source currents. Accordingly, the horizontal component of the ground magnetic field created by the telluric currents does not decrease 


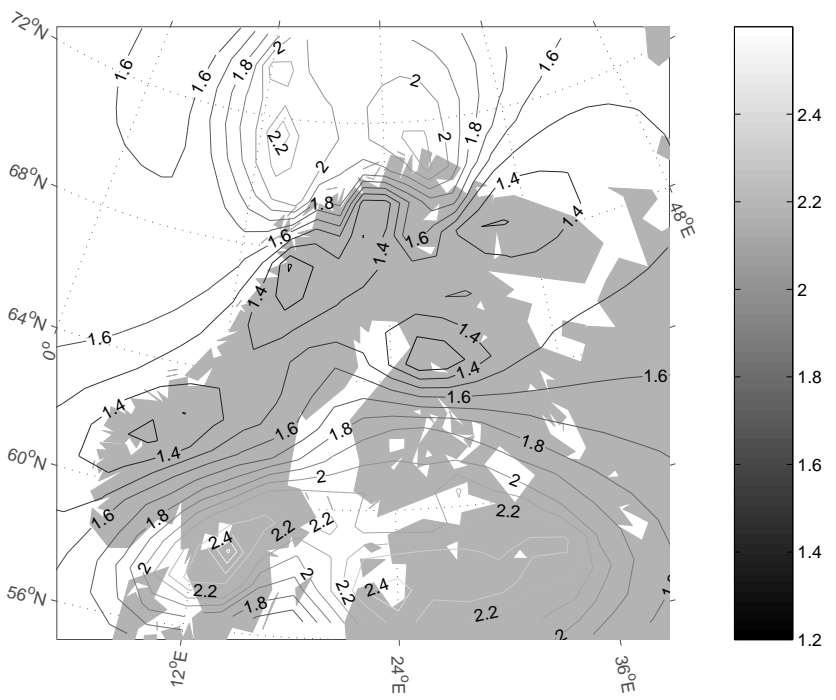

Fig. 11. $\log _{10}$ of the mean relative difference 100 $\left|\boldsymbol{J}_{\text {tot }}-\boldsymbol{J}_{\text {ext }}\right| /\left|\boldsymbol{J}_{\text {ext }}\right|$ taken over the entire modeling period.

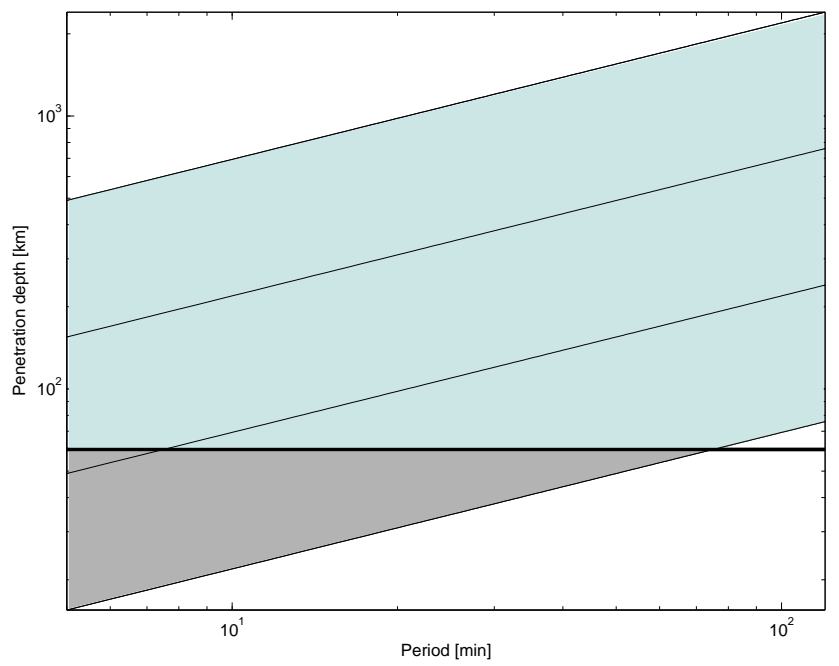

Fig. 12. Penetration depth of the periods from 5 to $120 \mathrm{~min}$ for average conductivities in Fig. 3. Thin lines correspond to conductivities $10^{-0.5}, 10^{-1.5}, 10^{-2.5}$ and $10^{-3.5} \mathrm{~S} / \mathrm{m}$ from the bottom to the top. The thick line indicates the $60 \mathrm{~km}$ penetration depth, i.e. the bottom of the 3 -D crust used in the modeling.

as rapidly as that of the ionospheric currents (for a more detailed discussion on this see, e.g. Tanskanen et al., 2001). Varentsov et al. (2002) found strong electromagnetic sounding parameter-related distortions caused by the same 3-D crust model. Thus, it is surprising that the overall equivalent current pattern is not very severely distorted in the region of the main current flow, i.e. gradients in Fig. 10 are relatively small. There are, however, features that clearly relate to inhomogeneities of the model, for example, the band of conductivity enhancement through the center parts of the model, as well as the currents induced in a well-conducting sea channel, as seen from Figs. 9 and 10. Also, the patch of the conductivity enhancement in northern Fennoscandia clearly creates distortions to the equivalent current patterns.

Features observed in Figs. 7-10 are also reproduced in the mean behavior of the relative difference taken over the whole modeling period depicted in Fig. 11. Excluding the impact of the complex 3-D structures in northern Fennoscandia, which cause some observable distortions even under the main current flow, the pattern-distorting effects of conductivity anomalies are seen even less in the mean values. The regions with a large relative difference correspond mainly to areas where the mean current amplitude is small. It is thus clear that for the investigated periods (5 to $120 \mathrm{~min}$ ), the strong inhomogeneities in the crust $(0-60 \mathrm{~km})$ affect the basic equivalent patterns relatively little, even during very dynamic events such as substorms.

The reason for the small effect of the 3-D crust structures is that the electromagnetic field, especially for the lower frequency portion of the external variations that comprise the majority of the observed signal, penetrates to depths greater than $60 \mathrm{~km}$. At these depths, in terms of the horizontal conductivity variations, the smoother upper mantle creates a spatially smooth response in the surface geomagnetic field. This is illustrated in Fig. 12, where the penetration depths for average conductivities of Fig. 3 for periods used in the modeling are shown. The light shading indicates the portion of the field penetrating below $60 \mathrm{~km}$, i.e. to depths having 1$\mathrm{D}$ conductivity, where dark shading, in turn, indicates the portion penetrating to less than $60 \mathrm{~km}$. As seen, the major part of the relatively low-frequency source field created by the varying ionospheric equivalent currents does not "see" the upper crust inhomogeneities because of the large depth to which it penetrates. Thus, spatially smooth induced fields are to follow. Furthermore, in comparison to the geoelectric response, the geomagnetic response "integrates" information from larger areas. This results in smoother overall field patterns, as seen by comparing Figs. 4 and 5 .

Based on the findings above, it is clear that in most of the ionospheric investigations the amplification of the determined current amplitudes, due to geomagnetic induction, is probably a more severe problem than the actual spatial distortion of the computed current patterns. This and the wide use of different indices derived from geomagnetic recordings encouraged us to seek a useful relation that could be used in estimating the internal part, for example, of the measured $I L$ index. The details of deriving such a relation are given in the Appendix; the final result is

$I L_{\text {int }}(\omega)=I L_{\text {tot }}(\omega) \frac{\alpha}{2(\alpha+p)}$,

where $I L_{t o t}$ is the measured signal, $\alpha=h+L$ is the sum of the height $h$ and $2 L$ half-width of the electrojet. For a homogeneous Earth we have

$p=\frac{1}{\sqrt{i \omega \mu_{0} \sigma}}$,

where $\omega, \mu_{0}$ and $\sigma$ are the angular frequency $(2 \pi f)$ of the variations, the vacuum permeability and the Earth's conductivity, respectively. To apply Eq. (1) one just needs to Fourier 
transform the measured signal, carry out the multiplication and invert the internal part back to the time domain.

Let us make a few observations from Eq. (1) that characterize some of the very basic features of geomagnetic induction. First, as the conductivity increases, the internal part also increases: for a perfectly conducting Earth, the internal part is exactly half of the measured signal. Secondly, the increasing width of the electrojet also increases the internal part: for $\alpha \gg|p|$, i.e. the source is planar, the internal part is again exactly half of the measured signal. Obviously, with $\omega=0$ there is no induction in the Earth and the internal part vanishes.

To test the new method, we separated the measured, 5min low-pass filtered $I L$ index for the 26 June 1998 event. An effective conductivity of $10^{-2} \mathrm{~S} / \mathrm{m}$ (and for comparison with conductivities $10^{-1} \mathrm{~S} / \mathrm{m}$ and $10^{-3} \mathrm{~S} / \mathrm{m}$ ) and $\alpha$ of $610 \mathrm{~km}$ were used. The choices are good estimates for the present situation, as can be verified from Figs. 3 and 7. To have the best possible correspondence with Fig. 6, only the continental IMAGE stations were used. The result is shown in Fig. 13. The similarity with Fig. 6 is clear: after the onset of the substorm, the internal part increases, reaches its maximum of about $30 \%$ at the end of the expansion phase after which a gradual decrease follows. Thus, although a more thorough analysis of the performance of Eq. (1) is needed, the simple method seems to produce a very realistic internal field for the 26 June 1998 substorm period. However, keeping the approximations made in deriving Eq. (1) in mind it should be emphasized that a reliable estimation of the internal part from the measured magnetic field variations requires 2-D array data and the application of rigorous magnetic field separation methods (see Pulkkinen et al., 2003b).

\section{Conclusions}

In this investigation, a combination of a field separation technique (Pulkkinen et al., 2003b) with a volume-3-D modeling of geomagnetic induction (Avdeev et al., 2002) was introduced. The combination was applied using a unique data set of geomagnetic recordings and a realistic model of the Earth's 3-D conductivity (Korja et al., 2002). The modeling results were used to study the impact of induction in the Fennoscandian region on the auroral ionospheric currents determined from the geomagnetic field recordings.

It was seen that despite the strong 3-D anomalies contained in the conductivity model, the overall spatial patterns of the determined ionospheric equivalent currents were not severely distorted. Although the effects of the 3-D induction were identified, the majority of the induced currents (for periods from 5 to $120 \mathrm{~min}$ ) flowed in the depths below the strong crustal conductivity anomalies, thus resulting in spatially relatively smooth, magnetic field variations. However, despite the small spatial effects of the 3-D conductivity, otherwise significant induction effects were observed. During the studied substorm onset, the amplitude of the current was overestimated by about $30 \%$. This is a number which can certainly affect, for example, estimations of the total ionospheric Joule
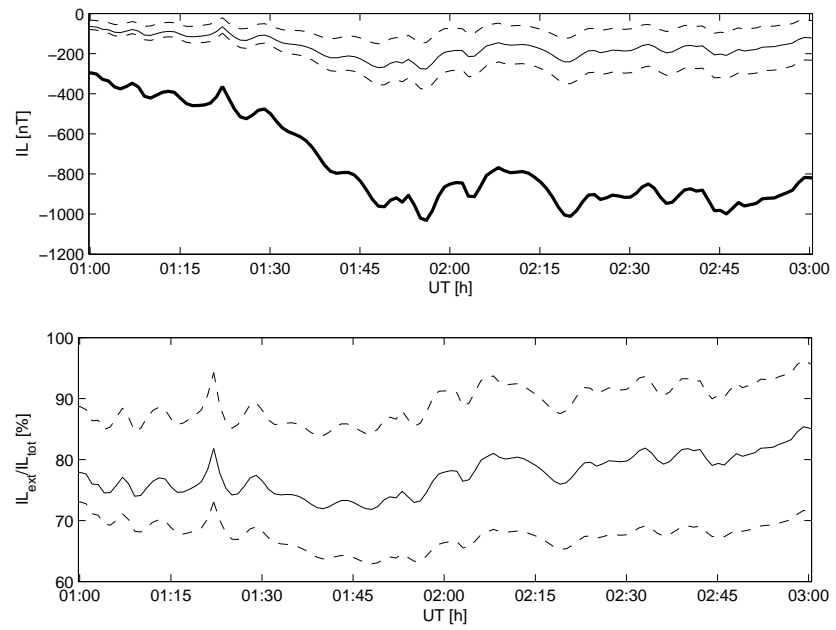

Fig. 13. Top panel: the measured total (thick solid) and the computed internal part of the 5-min low-pass filtered $I L$ index for the 26 June 1998 substorm event. Bottom panel: ratio between the external $I L$ and the total $I L$. Only continental IMAGE stations were used to compute the index. The dashed curves in both panels correspond to fields computed with conductivities $10^{-1} \mathrm{~S} / \mathrm{m}$ (lower curve) and $10^{-3} \mathrm{~S} / \mathrm{m}$ (upper curve), respectively. See the text for details.

heating and thus may also impact the estimations of the total magnetospheric energy budget (e.g. Tanskanen, 2002). Furthermore, the rapid increase of the internal part relative to the total magnetic field when moving away from the main ionospheric current flow was underscored. Accordingly, special care should be taken when interpreting data outside the main flow. In summary, though the spatial effects were found to be smaller than anticipated, it is clear that geomagnetic induction should be taken into account in detailed ionospheric investigations.

It should be noted that the conclusions made here are strictly applicable only for the source used and the conductivity model used, generalizations to other situations should be made with care. For example, the well-conducting sea is rather shallow in the western coast of Fennoscandia; steeper boundaries between deep seas and continents are more likely to also distort the spatial patterns of the computed ionospheric equivalent currents. Likewise, the study is strictly valid only for periods used of the field variations, i.e. periods from 5 to $120 \mathrm{~min}$. The higher-frequency electromagnetic fields which do not penetrate as deep may be distorted more severely by the 3-D conductivity anomalies in the crust. If the high-frequency component is not critical for a specific study, it may be worthwhile to low-pass filter the data to contain only frequencies that can be assumed to penetrate in the ground down to depths with smoother conductivity variations.

Motivated by the fact that the amplification effect dominates over the spatial effect, we, in addition to the described 3 -D modeling work, derived a simple relation that can be used to estimate the internal part of the measured $I L$, or similar index. Despite some very broad assumptions made 
in the derivation of the relation, application to IMAGE array geomagnetic data of the 26 June 1998 substorm event produced very reasonable results. Thus, especially under a well-defined, one-dimensional electrojet (no significant variations in the $y$-direction), with good approximate estimates of the effective ground conductivity and the electrojet width, the method can significantly improve the estimates of the external part in the $I L$ index variations.

\section{Appendix A}

Here the derivation of Eq. (1) is presented. The setting is as follows: 1 . We assume a one-dimensional, infinitely long electrojet at height $h$ having current distribution with the flow to the $y$-direction given by

$j_{y}(x)=\frac{I}{\pi} \frac{L}{x^{2}+L^{2}}$,

where $I$ is the current amplitude and $2 L$ is the half-width of the distribution. The magnetic field at the Earth's surface produced by the distribution in Eq. (A1) is equal to the field of a line current at height $h+L$ (Maurer and Theile, 1978). 2. We assume a homogeneous Earth with conductivity $\sigma$, and as usually in geomagnetic investigations, variations are assumed to be slow enough to justify the neglect of the displacement currents.

Using the complex image method, which is an extension of a classical method of images (e.g. Jackson, 1999, p.57), for an infinitely long line current (Boteler and Pirjola, 1998), we obtain an expression for the total magnetic field at the surface of the Earth directly below the line current $(x=0)$

$B_{x}^{t o t}(x=0)=\frac{\mu_{0} I}{2 \pi}\left(\frac{1}{\alpha}+\frac{1}{\alpha+2 p},\right)$,

where $\alpha=h+L$ and $p$ represent the complex depth of the image current which reduces for a homogeneous Earth to (Schmucker, 1970)

$p=\frac{1}{\sqrt{i \omega \mu_{0} \sigma}}$

where $\omega$ and $\mu_{0}$ are the angular frequency $(2 \pi f)$ of the variations and the vacuum permeability, respectively. Note that Eq. (A2) is valid for any layered Earth conductivity, if a general definition of the complex depth is used. The complex image approximation is generally valid when $|p v|^{3} \ll 1$ where $v$ is the wave number of the horizontal field variations (Thomson and Weaver, 1975; Boteler and Pirjola, 1998).

Now, the second term in the right-hand side of Eq. (A2) corresponds to the internal component produced by the image current at depth $h+L+2 p$, and thus the ratio between the internal and the total components of the magnetic field can be expressed as

$$
\frac{B_{x}^{i n t}}{B_{x}^{t o t}}=\frac{\alpha}{2(\alpha+p)}
$$

The $I L$ index is supposed, by definition, to measure the maximum excursion of the $x$-component of the magnetic field from the baseline level. Accordingly, if we assume a 1-D electrojet as described above, the value entering $I L$ is obtained directly below the center of the electrojet, i.e. $I L_{t o t}=B_{x}^{t o t}(x=0)$. Thus, we may interchange the two to give in the frequency domain

$$
I L_{\text {int }}(\omega)=I L_{\text {tot }}(\omega) \frac{\alpha}{2(\alpha+p)}
$$

which is the result we were looking for.

Acknowledgements. The research was performed while AP held a National Research Council Associateship Award at NASA/Goddard Space Flight Center. This investigation could not have been made without the great efforts of the BEAR project team responsible for part of the data used in the study at hand. The project was a good demonstration of how the knowledge coming from both the space physics and the solid earth communities can be combined for a new science. Invaluable comments by A. Viljanen and two referees on the manuscript are greatly acknowledged.

The Editor in Chief thanks A. Kuvshinov and another referee for their help in evaluating this paper.

\section{References}

Amm, O. and Viljanen, A.: Ionospheric disturbance magnetic field continuation from the ground to ionosphere using spherical elementary current systems, Earth, Planets and Space, 51, 431-440, 1999.

Avdeev, D. B., Kuvshinov, A. V., Pankratov, O. V., and Newman, O. G.: Three-dimensional induction logging problems, Part I: An integral eqution solution and model comparisons, Geophysics, 67(2), 413-426, 2002.

Beamish D., Clark, T. D. G., Clarke, E., and Thomson, A. W. P.: Geomagnetically induced currents in the UK: geomagnetic variations and surface electric fields. J. Atmos. Sol. Terr. Phys, 64, 1779-1792, 2002.

Berdichevsky, M. and Zhdanov, M,: Advanced theory of deep geomagnetic sounding, Elsevier Science Publishers B. V., Netherlands, 408, 1984.

Boteler, D. H. and Pirjola, R. J.: The complex-image method for calculating the magnetic and electric fields produced at the surface of the Earth by the auroral electrojet, Geophys. J. Int., 132(1), 31-40, 1998.

Engels, M., Korja, T., and BEAR Working Group: Multisheet modelling of the electrical conductivity structure in the Fennoscandian Shield, Earth Planets Space, 54, 559-573, 2002.

Heinson, G.: Electromagnetic studies of the lithosphere and asthenosphere, Surv. Geophys., 20, 229-255, 1999.

Häkkinen, L., Pulkkinen, T. I., Nevanlinna, H., Pirjola, R., and Tanskanen, E: Effects of induced currents on $D_{s t}$ and on magnetic variations at mid-latitude stations, J. Geophys. Res., 107, doi:10.1029/2001JA900130, 2002.

Jackson, D.: Classical Electrodynamics, 3rd ed., John Wiley and Sons, Inc., 1999.

Kaikkonen, P.: Thin-sheet modeling for deep electromagnetic studies in the Fennoscandian shield, Deep Electromagnetic Exploration, edited by: Roy, K. K., Sherma, S. K., and Mallick, K., Narossa Publishing House, 364-386, 1998. 
Kamide, Y. and Baumjohann, W.: Magnetosphere-ionosphere coupling, Physics and Chemistry in Space, 23, 178, 1993.

Kauristie, K., Pulkkinen, T. I., Pellinen, R. J., and Opgenoorth, H. J.: What can we tell about global auroral electrojet activity from a single meridional magnetometer chain?, Ann. Geophys., 14, 1177-1185, 1996,

http://direct.sref.org/1432-0576/ag/1996-14-1177

SRef-ID: 1432-0576/ag/1996-14-1177.

Korja, T., Engels, M., Zhamaletdinov, A. A., Kovtun, A. A., Palshin, N. A., Smirnov, M. Yu., Tokarev, A., Asming, V. E., Vanyan, L. L., Vardaniants I. L., and the BEAR Working Group: Crustal conductivity in Fennoscandia - a compilation of a database on crustal conductance in the Fennoscandian Shield, Earth, Planets and Space, 54, 535-558, 2002.

Mareschal, M.: Modelling of natural sources of magnetospheric origin in the interpretation of regional induction studies: a review, Surveys in Geophysics, 8, 261-300, 1986.

Maurer, H. and Theile, B.: Parameters of the Auroral Electrojet From Magnetic Variations Along a Meridian. J. Geophys., 44, 415-426, 1978.

Olsen, N. and Kuvshinov, A.: Modeling the ocean effect of geomagnetic storms, Earth Planets Space, 56, 525-530, 2004.

Pirjola, R.: Electromagnetic induction in the Earth by a plane wave or by fields of line currents harmonic in time and space, Geophysica, 18, 1982.

Pulkkinen, A., Amm, O., Viljanen, A., and BEAR Working Group: Ionospheric equivalent current distributions determined with the method of spherical elementary current systems, J. Geophys. Res., 108, doi:10.1029/2001JA005085, 2003a.
Pulkkinen, A., Amm, O., Viljanen, A., and BEAR Working Group: Separation of the geomagnetic variation field on the ground into external and internal parts using the spherical elementary current system method, Earth, Planets and Space, 55, 117-129, $2003 \mathrm{~b}$.

Schmucker, U.: Anomalies of geomagnetic variations in the southwestern United States, 13., Bull. Scripps, Institution of Oceanographie, University of California, 1970.

Tanskanen, E. I., Viljanen, A., Pulkkinen, T. I., Pirjola, R., Häkkinen, L., Pulkkinen, A., and Amm, O.: At substorm onset, $40 \%$ of AL comes from underground, J. Geophys. Res., 106, 13 119-13 134, 2001.

Tanskanen, E.: Terrestrial substorms as a part of global energy flow, $\mathrm{PhD}$ thesis, Finnish Meteorological Institute, Contributions, 36, 2002.

Thomson, D. J. and Weaver, J. T.: The complex image approximation for induction in a multilayered Earth, J. Geophys. Res., 80, 123-129, 1975.

Untiedt, J. and Baumjohann, W.: Studies of polar current systems using the IMS Scandinavian magnetometer array, Space Sci. Rev., 63, 245-390, 1993.

Varentsov, I., Engels, M., Korja, T., Smirnov, M., and the BEAR Working Group: A generalized model of Fennoscandia: a challenging database for long-period 3-D modeling studies within the Baltic Electromagnetic Array Research (BEAR) project, Izvestija, Phys. Solid Earth, 38, 855-896, 2002.

Viljanen, A., Kauristie, K., and Pajunpää, K.: On induction effects at EISCAT and IMAGE magnetometer stations, Geophys. J. Int., 121, 893-906, 1995.

Viljanen, A., Amm, O., and Pirjola, R.: Modeling geomagnetically induced currents during different ionospheric situations, J. Geophys. Res., 104, 28 059-28 072, 1999. 